\title{
Cutaneous larva MIGRANS - a threat tO diVERS IN THE TROPICS
}

Romuald Olszański, Piotr Siermontowski, Zbigniew Dąbrowiecki

Maritime \& Hyperbaric Medicine Department, Military Institute of Medicine, Gdynia

\section{ABSTRACT}

The article presents a dermatosis that occurs in tropical and subtropical countries. Though the definitive hosts of the cutaneous larva migrans parasite are animals, humans can become accidental hosts and they are infected when their skin comes into contact with damp soil, most frequently sand. The disease is only present in the epidermis where an itch is brought about by the mining activity of the larva. Sunbathers and divers who put on their gear on a beach, on account of the epidermis maceration caused by a prolonged exposure to water, are particularly susceptible to the penetrative activities of the larva. In Poland the cutaneous larva migrans is in most cases mistaken for nettle rash or eczema.

Key words: tropics, parasite, beach, diving.

ARTICLE INFO

PolHypRes 2016 Vol. 56 Issue 3 pp. 33 - 36

ISSN: $1734-7009$ elSSN: 2084-0535

DOI: $10.1515 / p h r-2016-0017$

Pages: 4, figures: 2 , tables: 0

page www of the periodical: www.phr.net.pl

Publisher

Polish Hyperbaric Medicine and Technology Society

\section{Informing article}

Delivery date: $02.02 .2016 r$.

Date of approval for print: $25.09 .2016 r$. 


\section{INTRODUCTION}

The cutaneous larva migrans, CLM, otherwise known as 'migrant linear epidermitis', 'beach worm', 'migrant helminthiasis', 'dermatitis serpiginous', 'creeping eruption' or 'sandworm' is a tropical dermatosis caused by the larvae of the alimentary canal parasites in animals.

The cutaneous larva migrans occurs in tropical and subtropical areas in South America, Africa, Caribbean, South-East Asia, the southern states of the USA and the Mediterranean Basin [1]. It is the most frequently occurring dermatosis in tourists returning from the tropics [2].

The etiological factor of the cutaneous larva migrans chiefly includes the nematode larvae of canines and felines: Ancylostoma caninum - canines, Ancylostoma braziliense - canines and felines. CLM can be also caused by other canine and feline larvae: Uncinaria stenocephala, Ancylostoma tubaeforme, Gnathostoma spinigerum, Strongyloides stercoralis, bovine parasites Bunostomum phlebotomum, rodent parasites Strongyloides myopotami and Strongyloides procyonis.

Nematode eggs enter the soil together with the faeces of infected animals and then, in the hot and humid climate, the larvae are hatched [3]. The disease is only present in the epidermis as larvae do not secrete collagenase which is prerequisite for the transition through the basement membrane of the epidermis [4].

The most exposed groups in tropical countries are farmers, gardeners, plumbers tourists and divers.

\section{Clinical DesCRiption}

The incubation period of the disease spans from 5 to 15 days from infection [2]. Once infected, each larva daily bores a 2-3 cm long meandering tunnel in the epidermis, which induces a strong itch [2]. The first symptom is an itchy blister then followed by itchy, restiform, winding, towering redness up to between ten and twenty centimetres long, ended with a lump or a blister - where the parasite is found. Larvae in humans do not transform into adult forms but they die after weeks or months of 'straying' inside the epidermis, e.g. Ancylostoma braziliensis can live in the epidermis up to 200 days. Upon the infection larvae can remain dormant and asymptomatic in skin for several weeks or months [5].

The disease most commonly occurs in tourists strolling barefooted or sunbathing on sand, or putting on diving gear on a beach in the tropics. For that reason the lesions most commonly occur on the feet (fig. 1), lower legs, thighs, buttocks and forearms (fig. 2). Occasionally, symptoms might present themselves in unusual places, e.g. the back [3], or the scalp [6].

The diagnosis reveals peripheral eosinophilia and the increased level of immunoglobulin E. A detection of the cutaneous larva migrans in the epidermis is aided by dermoscopy [2].

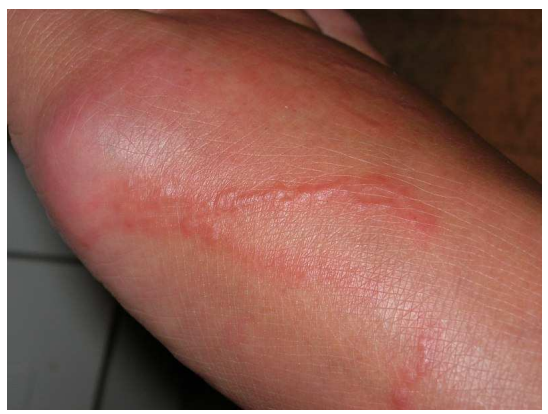

Fig. 1. Cutaneous larva in the sole part of the foot

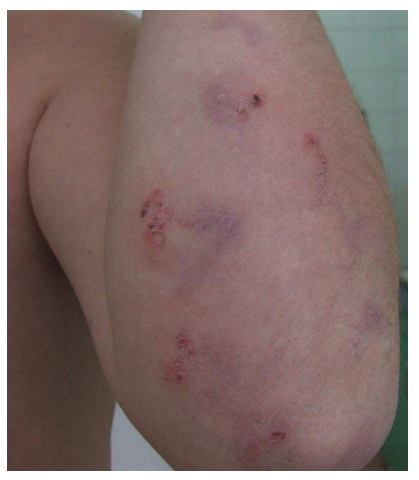

Fig. 2. Cutaneous larva in the forearm area. 
The primary treatment consists in a single dose of Ivermectin $200 \mu \mathrm{g} / \mathrm{kg}$ m.c. or Albendazole $400 \mathrm{mg}$ taken daily for 3 to 7 days. Locally: cryotherapy or ointment with ivermectin [7].

The cutaneous larva migrans in Poland and other European countries [8] is most often mistakenly diagnosed as nettle rash (55\%). Mistaken diagnosis and the following badly administered treatment of the cutaneous larva migrans can be applied to $22 \%$ to $58 \%$ cases globally [9].

Pictures of divers infected with the cutaneous larva migrans have been demonstrated. Polish doctors diagnosed it as a nettle rash or eczema and ineffectively treated it for several days with antihistamines and locally with steroids.

\section{BIBLIOGRAPHY}

1. Reichert F, Pilger D, Schuster A, Lesshafft H, Guedes de Oliveira S, Ignatius R, Feldmeier H. Prevalence and Risk Factors of HookwormRelated Cutaneous Larva Migrans (HrCLM) in a Resource-Poor Community in Manaus, Brazil. PLOS Neglected Tropical Diseases 24: 1-13, 2016;

2. Vasievich MP, Villarreal JDM, Tomecki KJ. Got the Travel Bug? A Review of Common Infections, Infestations, Bites, and Stings Among Returning Travelers. Am J Clin Dermatol, 10.1007/s40257-016-0203-7, 2016;

3. Comparin C, Rodrigues MM, Santos BC Images in Clinical Tropical Medicine Extensive Cutaneous Larva Migrans with Eczematous Reaction on Atypical Localization Am. J. Trop. Med. Hyg., 94(6),1185-1186, 2016;

4. Ma DL, Vano-Galvan S. Creeping Eruption - Cutaneous Larva Migrans NEngl J Med 374;14, 2016.

5. Belizario Jr V, Trinos JPC, Garcia NB, Reyes M. Cutaneous Manifestations of Selected Parasitic Infections in Western Pacific and Southeast Asian Regions. Curr Infect Dis Rep 18: 30, 1-7, 2016

6. Meotti CD. Cutaneous larva migrans on the scalp: atypical presentation of a common disease An Bras Dermatol. 89(2):332-3, 2014;

7. Fischer $S$, Nenoff $P$. Cutaneous larva migrans: successful topical treatment with ivermectin - a case report. Deutsche Dermatologische Gesellschaft (DDG) 1610-0379/140, 2016;

8. Olszański R. Problemy zdrowotne w tropiku [Health issues in the tropics] WIM Warsaw 2014

9. Neils Ben Quashie NB, Tsegah E. An unusual recurrence of pruritic creeping eruption after treatment of cutaneous larva migrans in an adult Ghanaian male: a case report with a brief review of literature. Pan African Medical Journal. 21:285, 2015.

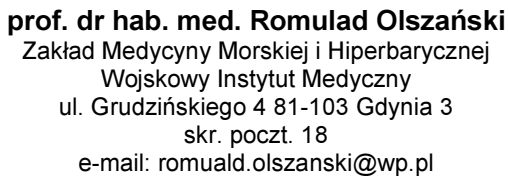

